\title{
Flow of Soap Films on Inclined Plane
}

Joseph G. Abdulahad

College of Education

University of Duhok

\section{Received on: 13/9/2009}

\section{ABSTRACT}

Accepted on: 12/4/2010

In this paper a mathematical model is constructed to describe a two dimensional flow for an inclined films with an inclination angle $\alpha$ to the horizontal that is drainage under the action of gravity. An asymptotic analysis is employed with the use of lubrication approximation. The film is assumed to be supported by wire frame elements at the ends. We apply the Navier-Stokes equations for flow of an incompressible fluid in two dimensions with specified boundary conditions. We obtain the equations representing the film thickness, the surface concentration, and the surface velocity. We obtain the similarity solutions for extensional flow of the simplified forms of these equations by using Lagrangian coordinate and then we approximate this equation by using Taylor series to obtain another similarity equation that can be used for different values of time.

Keywords: Flow of films, Navier - Stokes equations, incompressible fluid, boundary conditions.

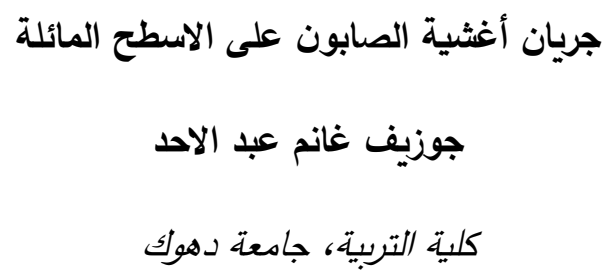

تاريخ القبول: 2010/04/12

تاريخ الاستلام: 2009/09/13

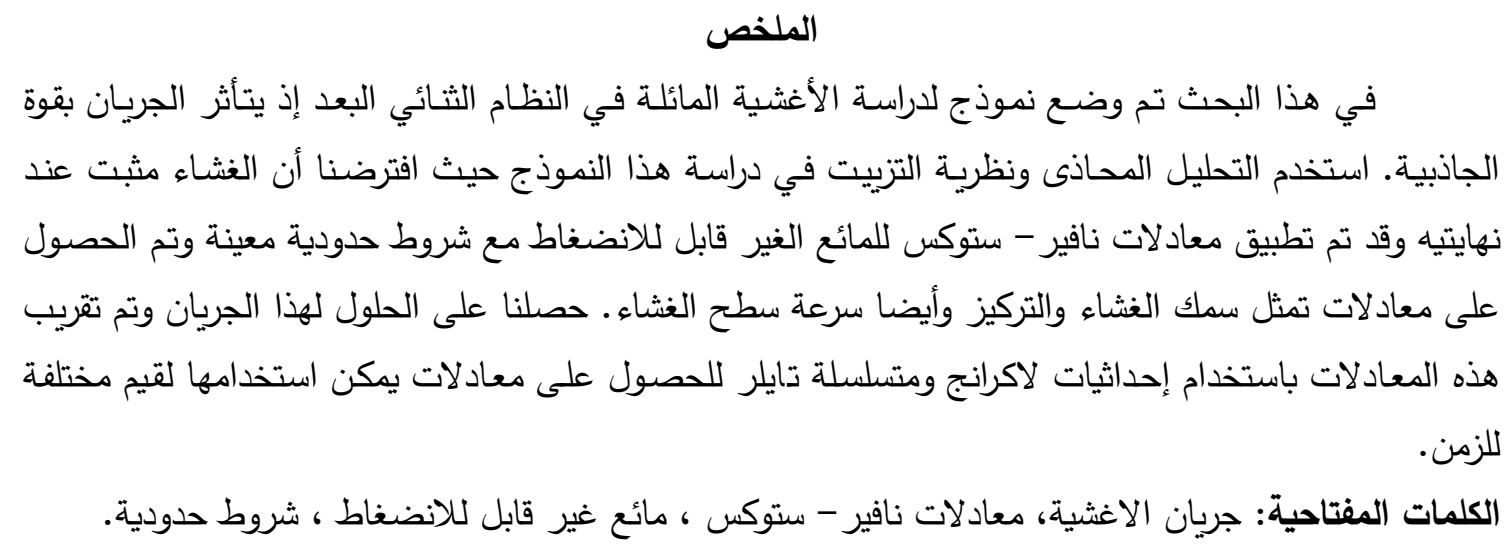

\section{Introduction:}

Thin films have been studied widely in many areas, such as surface coatings in paint, varnish and silver layer on a compact disc. The drainage of thin film is important in understanding foam fabrications for applications like making cushions and these foam are not aqueous. There are a variety of phenomena one can observe such as drainage, details of rapture [1] and these phenomena can help to characterize and describe the physical processes that occur in our real world and such knowledge can be used in surface coatings in paint, protective wax and foam development [2]. 
The drainage of soap films has been studied by many authors. Mysel [7] gave a comprehensive experimental description of soap films thinning. The theoretical and numerical model for soap film drainage studied by Schwartz [8] which reproduce may of the features of this process that have been observed in experiment. These features include the shape of the film thickness profile when the film is taken to be vertical and supported by wires, and the large differences in drainage time scales for low and high surfactant. Myers [5, 6] investigated the driven of thin film flow when the surface tension play an important role. Kondic, L.[3] studied the instabilities in gravity driven flow of thin liquid films. The main purpose of this paper is to study the inclined drainage flow in mobile and immobile films in which the surfactant forces play an important role on the drainage of thin liquid films.

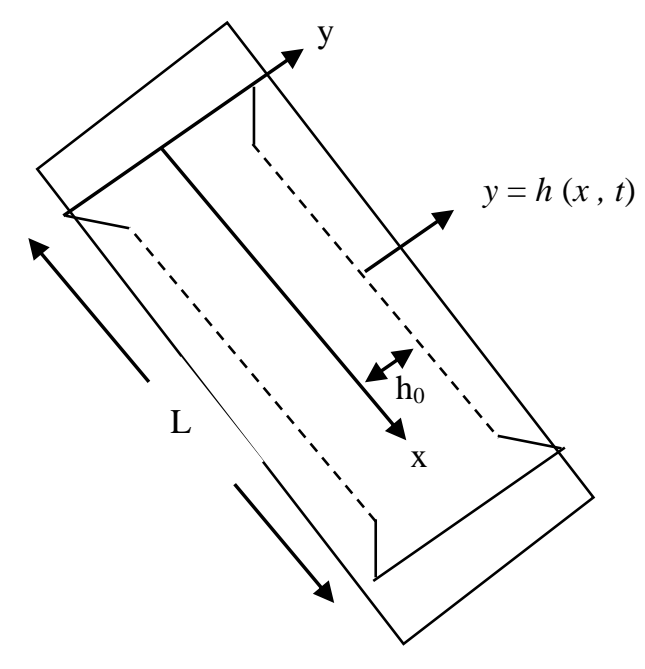

Figure (1). Schematic diagram for an inclined surface liquid film flow

\section{Formulations and Governing Equations:}

We consider an inclined two dimensional soap film supported at the ends by guides. The liquid air interface is located at $y= \pm h(x, t)$ where the film is symmetric with respect to the central line $y=0$ as shown in figure (1) and we consider only the half film $h(x, t) \geq 0$ and the model is characterized by a very small Reynolds number. Let $U(u, v)$ be the fluid velocity, where $u$ and $v$ represent the velocity components in $x$ and $y$ directions respectively.

The continuity equation is given by

$$
\frac{\partial u}{\partial x}+\frac{\partial v}{\partial y}=0
$$

and the momentum equations in $x$ and $y$ directions are respectively given by:

$P\left(\frac{\partial u}{\partial t}+u \frac{\partial u}{\partial x}+v \frac{\partial u}{\partial y}\right)=-\frac{\partial P}{\partial x}+\mu\left(\frac{\partial^{2} u}{\partial x^{2}}+\frac{\partial^{2} u}{\partial y^{2}}\right)+\rho g \sin \alpha$

and 
$P\left(\frac{\partial v}{\partial t}+u \frac{\partial v}{\partial x}+v \frac{\partial v}{\partial y}\right)=-\frac{\partial P}{\partial y}+\mu\left(\frac{\partial^{2} v}{\partial x^{2}}+\frac{\partial^{2} v}{\partial y^{2}}\right)-\rho g \cos \alpha$

Which hold within the fluid half layer $0 \leq y \leq h(x, t)$ and where $P$ and $\mu$ represent density and viscosity of fluid, $g$ is the gravity and $t$ the time.

The boundary conditions to be imposed are as follows:

Since the film is symmetric, we have

at $y=0, v=0$

and

at $y=0, \frac{\partial u}{\partial y}=0$

Furthermore at the surface of the film, the kinematics condition is given by:

$\frac{\partial h}{\partial t} \equiv v-u \frac{\partial h}{\partial x}$

Also the shear stress and normal stress conditions on the free surface of the film are respectively given by:

$\tau(x, t)=\mu\left(\frac{\partial u}{\partial y}+\frac{\partial v}{\partial x}\right)+2 \mu \frac{\partial h}{\partial x}\left(\frac{\partial v}{\partial y}-\frac{\partial u}{\partial x}\right)$

and

$P_{s}=-P-2 \mu\left[\frac{\partial h}{\partial x}\left(\frac{\partial u}{\partial y}-\frac{\partial v}{\partial x}\right)+\frac{\partial v}{\partial y}\right]$

Where $P_{s}$ is the pressure at the liquid side of the free surface. We have to note here that since $\frac{\partial h}{\partial x}<1$ so we can neglect $\left(\frac{\partial h}{\partial x}\right)^{2}$.

Now we subdivide the velocity component $u$ of the flow field into a purely slug flow contribution $u_{0}=u_{0}(x, t)$ and a shear component $u_{1}(x, y, t)$ which satisfies the noslip condition at the free surface, that is at $y=h, u_{1}(x, y, t)=0$ and thus

$u(x, y, t)=u_{0}(x, t)+u_{1}(x, y, t)$

Now (2.1) and (2.9) give

$\frac{\partial u_{0}}{\partial x}+\frac{\partial u_{1}}{\partial x}+\frac{\partial v}{\partial y}=0$

Since the Reynolds number is taken so small, the inertia term in the Navier Stokes equation can be neglected in comparison with viscous forces per unit volume of the fluid and so equations (2) are reduced to give

$\frac{\partial P}{\partial x}=\mu\left(\frac{\partial^{2} u}{\partial x^{2}}+\frac{\partial^{2} u}{\partial y^{2}}\right)+\rho g \sin \alpha$

and

$\frac{\partial P}{\partial y}=\mu\left(\frac{\partial^{2} v}{\partial x^{2}}+\frac{\partial^{2} v}{\partial y^{2}}\right)-\rho g \cos \alpha$

By using equation (9), equations (7), (8) and (11), become respectively

$\tau(x, t)=\mu\left(\frac{\partial u_{1}}{\partial y}+\frac{\partial v}{\partial x}\right)+2 \mu \frac{\partial h}{\partial x}\left(\frac{\partial v}{\partial y}-\frac{\partial u_{0}}{\partial x}-\frac{\partial u_{1}}{\partial x}\right)$ 
$P_{s}=-P-2 \mu\left[\frac{\partial h}{\partial x}\left(\frac{\partial u_{1}}{\partial y}-\frac{\partial v}{\partial x}\right)+\frac{\partial v}{\partial y}\right]$

and

$\frac{\partial P}{\partial x}=\mu\left(\frac{\partial^{2} u_{0}}{\partial x^{2}}+\frac{\partial^{2} u_{1}}{\partial x^{2}}+\frac{\partial^{2} u_{1}}{\partial y^{2}}\right)+\rho g \sin \alpha$

Scaling and dimensional analysis:

If $L$ denotes the characteristic length scale and a typical scale for width is $h_{0}=\in L, \in<<1$, then the velocity scale is $V_{1}=\epsilon^{2} v_{0}$, where is $\mathrm{V}_{1}$ the sheer scale and $V_{0}$ is the extensional scale and the transverse velocity scale is $V=\in V_{0}$ and the pressure scale is $P=\mu V_{0} / L$.

We introduce the following non-dimensional variables as follows.

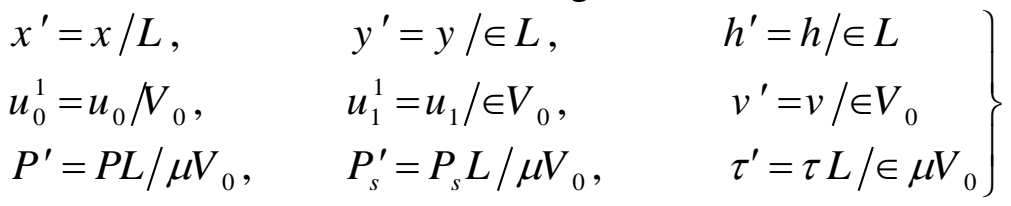

We define the Bond number $(B)$ and the capillary number $C a$ as

$B=L^{2} P g / \sigma$ and $C a=\mu V_{0} / \sigma$

where $\sigma:$ is the surface tension.

By using (16), equations (10), (12) and (15) become non-dimensional, dropping all primes for dimensionless variables, we obtain.

$\frac{\partial u_{0}}{\partial x}+\epsilon^{2} \frac{\partial u_{1}}{\partial x}+\frac{\partial v}{\partial y}=0$

$\frac{\partial P}{\partial y}=\epsilon^{2} \frac{\partial^{2} v}{\partial x^{2}}+\frac{\partial^{2} v}{\partial y^{2}}-\frac{\in B}{c a} \cos \alpha$

$\frac{\partial P}{\partial x}=\frac{\partial^{2} u_{0}}{\partial x^{2}}+\epsilon^{2} \frac{\partial^{2} u_{1}}{\partial x^{2}}+\frac{\partial^{2} u_{1}}{\partial y^{2}}+\frac{B}{c a} \sin \alpha$

Furthermore, the boundary conditions (4), (5), (13) and (14) become

$v=0, \frac{\partial u_{1}}{\partial y}=0$ at $y=0$

$\tau(x, t)=\left(\frac{\partial u_{1}}{\partial y}+\frac{\partial v}{\partial x}\right)+2 \frac{\partial h}{\partial x}\left(\frac{\partial v}{\partial y}-\frac{\partial u_{0}}{\partial x}-\in \frac{\partial u_{1}}{\partial x}\right)$

$P_{s}=-P-2 \in^{2} \frac{\partial h}{\partial x}\left(\frac{\partial u_{1}}{\partial x}+\frac{\partial v}{\partial x}\right)-2 \frac{\partial v}{\partial y}$

Lubrication model:

We expand each of the unknowns $u_{1}, v$ and $P$ as a power series in $\in$ as follows:

$\left.\begin{array}{l}u_{1}(x, y, t, \in)=u_{10}+\epsilon^{2} u_{11}+\ldots \\ v(x, y, t, \in)=v_{0}+\epsilon^{2} v_{1}+\ldots \\ P(x, y, t, \in)=P_{0}+\epsilon^{2} P_{1}+\ldots\end{array}\right\}$

The continuity equation (17) gives: 


$$
\begin{aligned}
& \frac{\partial u_{0}}{\partial x}+\epsilon^{2}\left(\frac{\partial u_{10}}{\partial x}+\epsilon^{2} \frac{\partial u_{11}}{\partial x}+\cdots\right)+\left(\frac{\partial v_{0}}{\partial y}+\epsilon^{2} \frac{\partial v_{1}}{\partial y}+\cdots\right)=0 \\
& \text { or } \\
& \left(\frac{\partial u_{0}}{\partial x}+\frac{\partial v_{0}}{\partial y}\right)+\epsilon^{2}\left(\frac{\partial u_{10}}{\partial x}+\frac{\partial v_{1}}{\partial y}\right)+\cdots=0
\end{aligned}
$$

By equating the powers of $\in$, we get:

$$
\left(\frac{\partial u_{0}}{\partial x}+\frac{\partial v_{0}}{\partial y}\right)=0
$$

Integrating (24) with respect to $y$ we have:

$v_{0}=-\frac{\partial u_{0}}{\partial x} y+c(x, t)$

Since at $y=0$, we have $v_{0}=0$, we get $c(x, t)=0$ and equation (25) reduces to give:

$v_{0}=-\frac{\partial u_{0}}{\partial x} y$

Also equation (18) reduces to give

$\frac{\partial P_{0}}{\partial x}=\frac{\partial^{2} u_{0}}{\partial x^{2}}+\frac{\partial^{2} u_{10}}{\partial y^{2}}+\frac{B}{C a} \sin \alpha$

and

$\frac{\partial P_{1}}{\partial x}-\frac{\partial^{2} u_{10}}{\partial x^{2}}-\frac{\partial^{2} u_{11}}{\partial y^{2}}=0$

and so on. Integrating both sides of equation (27) with respect to $y$ and since each of the functions $p_{0}$ and $u_{0}$ are functions of $x$ and $t$ only, we get

$\frac{\partial u_{10}}{\partial y}=\left(\frac{\partial P_{0}}{\partial x}-\frac{\partial^{2} u_{0}}{\partial x^{2}}-\frac{B}{C a} \sin \alpha\right) y+k_{1}(x, t)$

Since at $y=0$, we have $\frac{\partial u_{10}}{\partial y}=0$, we get $k_{1}(x, t)=0$ and equation (29) reduces to give

$\frac{\partial u_{10}}{\partial y}=\left(\frac{\partial P_{0}}{\partial x}-\frac{\partial^{2} u_{0}}{\partial x^{2}}-\frac{B}{C a} \sin \alpha\right) y$

equation (30) can be integrated to give

$u_{10}=\left(\frac{\partial P_{0}}{\partial x}-\frac{\partial^{2} u_{0}}{\partial x^{2}}-\frac{B}{C a} \sin \alpha\right) \frac{y^{2}}{2}+k_{2}(x, t)$

at $y=h, u_{10}=0$ and so from (31), we get

$k_{2}(x, t)=-\left(\frac{\partial P_{0}}{\partial x}-\frac{\partial^{2} u_{0}}{\partial x^{2}}-\frac{B}{C a} \sin \alpha\right) \frac{h^{2}}{2}$

Thus equation (31) give

$u_{10}=\frac{1}{2}\left(\frac{\partial P_{0}}{\partial x}-\frac{\partial^{2} u_{0}}{\partial x^{2}}-\frac{B}{C a} \sin \alpha\right)\left(y^{2}-h^{2}\right)$

Now from the boundary condition (29) and the expansion (23), we have

$P_{0}=2 \frac{\partial v_{0}}{\partial y}+P_{s}$ 
Using (24) in (33), we get

$P_{0}=-2 \frac{\partial u_{0}}{\partial x}+P_{s}$

or

$\frac{\partial p_{0}}{\partial x}=-2 \frac{\partial^{2} u_{0}}{\partial x^{2}}+\frac{\partial P_{s}}{\partial x}$

Substituting equation (34) into (30) and (32), we get respectively

$\frac{\partial u_{10}}{\partial y}=\left(-3 \frac{\partial^{2} u_{0}}{\partial x^{2}}+\frac{\partial P_{s}}{\partial x}-\frac{B}{C a} \sin \alpha\right) y$

and

$u_{10}=\frac{1}{2}\left(-3 \frac{\partial^{2} u_{0}}{\partial x^{2}}+\frac{\partial P_{s}}{\partial x}-\frac{B}{C a} \sin \alpha\right)\left(y^{2}-h^{2}\right)$

Which represent the shear velocity equation.

Also from (23), equation (19) gives

$-\frac{\partial P_{0}}{\partial y}+\frac{\partial^{2} v_{0}}{\partial y^{2}}-\frac{\in B}{C a} \cos \alpha+\epsilon^{2}\left(-\frac{\partial P_{1}}{\partial y}+\frac{\partial^{2} v_{0}}{\partial x^{2}}+\frac{\partial^{2} v_{1}}{\partial y^{2}}\right)+\cdots=0$

Which given

$\frac{\partial P_{0}}{\partial y}=\frac{\partial^{2} v_{0}}{\partial y^{2}}-\frac{\in B}{C a} \cos \alpha$

From (21), the shear stress condition, gives:

$$
\begin{aligned}
& \left(\frac{\partial u_{10}}{\partial y}+\epsilon^{2} \frac{\partial u_{11}}{\partial y}+\cdots\right)+\left(\frac{\partial v_{0}}{\partial x}+\epsilon^{2} \frac{\partial v_{1}}{\partial x}+\cdots\right)+2 \frac{\partial h}{\partial x}\left[\left(\frac{\partial v_{0}}{\partial y}+\epsilon^{2} \frac{\partial v_{1}}{\partial y}+\cdots\right)\right. \\
& \left.-\frac{\partial u_{0}}{\partial y}-\epsilon\left(\frac{\partial u_{10}}{\partial x}+\epsilon^{2} \frac{\partial u_{11}}{\partial x}+\cdots\right)\right]-\tau(x, t)=0+0 \epsilon^{2}+\cdots
\end{aligned}
$$

Comparing the similar terms of both sides of (38) in power of $\in$, we obtain

$$
\tau(x, t)=\left(\frac{\partial u_{10}}{\partial y}+\frac{\partial v_{0}}{\partial x}\right)+2 \frac{\partial h}{\partial x}\left(\frac{\partial v_{0}}{\partial y}-\frac{\partial u_{0}}{\partial x}\right)
$$

Using equation (24), (26) and (35), equation (39) on the free surface $y=h$, gives

$$
\tau(x, t)=\left(-4 \frac{\partial^{2} u_{0}}{\partial x^{2}}+\frac{\partial P_{s}}{\partial x}-\frac{B}{C a} \sin \alpha\right) h-4 \frac{\partial h}{\partial x} \frac{\partial u_{0}}{\partial x}
$$

or

$$
\tau(x, t)=-4 \frac{\partial}{\partial x}\left(h \frac{\partial u_{0}}{\partial x}\right)+h \frac{\partial P_{s}}{\partial x}-\frac{B}{C a} h \sin \alpha
$$

We have to noticed that the term $\frac{\partial^{2} u_{0}}{\partial x^{2}}$ can be neglected since it is of order $\in^{2}$ and it is smaller than the term $u_{0}$.

Evolution equation of the film thickness:

It is known that the conservation of mass in integral form is

$$
\frac{\partial h}{\partial t}=-\frac{\partial \varphi}{\partial x}=-\frac{\partial}{\partial x} \int_{0}^{h} u(x, y, t) d y
$$

Where $\varphi$ is the volumetric flow rate. 
From (19) and (23), equation (41), gives

$\frac{\partial h}{\partial t}=-\frac{\partial}{\partial x} \int_{0}^{h}\left(u_{0}+u_{10}+\epsilon^{2} u_{11}\right) d y$

Using equation (36), equation (42) after simplification becomes

$\frac{\partial h}{\partial t}=-\frac{\partial}{\partial x}\left[\left(u_{0} h\right)-\frac{\epsilon^{2} h^{3}}{3} \frac{\partial P_{s}}{\partial x}+\frac{\epsilon^{2} h^{3}}{3} \frac{B}{C a} \sin \alpha\right]$

equation (43) is the evolution equation of the film thickness in non-dimensional form.

In dimensional form equation (40) and (43) are respectively

$\tau(x, t)=-4 \mu \frac{\partial}{\partial x}\left(h \frac{\partial u_{0}}{\partial x}\right)+h \frac{\partial P_{s}}{\partial x}-\rho g h \sin \alpha$

and

$\frac{\partial h}{\partial t}=\frac{-\partial}{\partial x}\left(u_{0} h-\frac{1}{3 \mu} h^{3} \frac{\partial P_{s}}{\partial x}+\frac{\rho g}{3 \mu} h^{3} \sin \alpha\right)$

The pressure $P_{s}$ in dimensional variables is defined as

$$
P_{s}=-\sigma k_{1}=-\sigma \frac{\partial^{2} h / \partial x^{2}}{\left[1+\left(\frac{\partial h}{\partial x}\right)^{2}\right]^{\frac{3}{2}}}
$$

Where $k_{1}$ is the surface curvature.

Surfactant effect:

A convection - diffusion evolution equation for an insoluble surfactant for a film of small slop is given by:

$\frac{\partial \Gamma}{\partial t}=-\frac{\partial}{\partial x}\left(u_{0} \Gamma\right)+D \frac{\partial^{2} \Gamma}{\partial x^{2}}$

Where $\Gamma(x, t)$ is a local surfactant concentration and $D$ is Fickian diffusion constant.

The simplest form for small change in surface tension is given by:

$\sigma(\Gamma)=\sigma_{0}-k\left(\Gamma-\Gamma_{0}\right)$

where $k_{1}$ is constant and the zero subscript refer to initial values of these quantities at the start of motion.

The surface shear stress is given by:

$\tau(x, t)=\frac{\partial \sigma}{\partial x}=-k \frac{\partial \Gamma}{\partial x}$

substituting (48) into equation (44), we get

$4 \mu \frac{\partial}{\partial x}\left(h \frac{\partial u_{0}}{\partial x}\right)=k \frac{\partial \Gamma}{\partial x}-\sigma_{0} h \frac{\partial^{3} h}{\partial x^{3}}-\rho g h \sin \alpha$

Furthermore equation (45), gives

$\frac{\partial h}{\partial t}=-\frac{\partial}{\partial x}\left(u_{0} h\right)-\frac{\sigma}{3 \mu} \frac{\partial}{\partial x}\left(h^{3} \frac{\partial^{3} h}{\partial x^{3}}\right)+\frac{\rho g}{3 \mu} \frac{\partial}{\partial x}\left(h^{3}\right) \sin \alpha$

we have to noticed that equations (46), (49) and (50) form a complete set.

Sample calculation:

Assume that $L=1 \mathrm{~cm}$ and $h_{0}$ must be less than $L$, we take $h_{0}=0.5$. The density of water $p=1 \mathrm{gm} / \mathrm{cm}^{3}$ and the surface tension $\sigma=72 d y n e / \mathrm{cm}$ and it can be reduced 
because of the existence of the surfactant, we take the viscosity $\mu=0.01$ poises. The input dimensionless parameters are formed from the physical values solution we take $B_{1}=0.2725$ and $C a=30000$. Profiles exhibit a concave-out form since the extensional flow that occurs before surface tension and surface tension gradient effects become important.

The early time solution is determined primarily by the simplified evolution equation (50) which gives the form

$\frac{\partial h}{\partial t}=-\frac{\partial}{\partial x}\left(u_{0} h\right)$

and the simplified form of force balance equation gives

$\frac{D h}{D t} \equiv \frac{\partial h}{\partial t}+u_{0} \frac{\partial h}{\partial t}=-h \frac{\partial u_{\circ}}{\partial x}$

and

$\frac{\partial}{\partial x}\left(h \frac{\partial u_{0}}{\partial x}\right)=-\frac{\rho g \sin \alpha}{4 \mu} h$

Integrating equation (52) with respect to $x$, we get

$h \frac{\partial u_{0}}{\partial x}=-\frac{\rho g \sin \alpha}{4 \mu} \int_{x}^{x(t)} h d x$

Combining equations (51) and (53), we get

$\frac{D h}{D t}=-\frac{\rho g \sin \alpha}{4 \mu} \int_{x}^{x(t)} h d x$

Now we solve the problem by using Lagrangian-discription of motion and we consider an initially uniform film $h(x, 0)=h_{0}, 0 \leq x \leq L_{0}$, where the evolution equation is determined by equations (51), (52) and (53).

Let $\checkmark$ be the initial position of a particle on the film that moves to position $x$ at a later time, that is

$x=x(\varsigma, t)$ and $x(\varsigma, 0)=\varsigma_{1}$

and so equation (54) reduces to gives

$\frac{D h}{D t}=-\frac{\rho g \sin \alpha}{4 \mu} \int_{x}^{\varsigma_{1}(t)} h(x,(\varsigma, 0)) d x=\frac{\rho g \sin \alpha}{4 \mu} h_{0}\left[\varsigma_{1}(t)-\varsigma\right]$

where $\varsigma_{1}(t)$ is a function that will be determined by the lower end boundary condition.

The derivative following the motion in the Lagrangian system is given by

$\frac{\partial h}{\partial t}=\frac{-\rho g \sin \alpha}{4 \mu} h_{0}\left[\zeta_{1}(t)-\zeta\right]$

and following the motion, the mass conservation condition requires that

$h=h_{0} \frac{\partial \varsigma}{\partial x}$

Thus equations (56) and (57) gives

$\frac{\partial}{\partial t}\left(h_{0} \frac{\partial \varsigma}{\partial x}\right)=-\frac{\rho g \sin \alpha}{4 \mu} h_{0}\left[\varsigma_{1}(t)-\varsigma\right]$

integrating equation (58), we get

$\left.\frac{\partial \varsigma}{\partial x}\right]_{0}^{t}=-\frac{\rho g \sin \alpha}{4 \mu}\left[\int_{0}^{t} \varsigma_{1}(t) d t-\varsigma t\right]$ 
since at $t=0, \frac{\partial \varsigma}{\partial x}=0$, so equation (59), gives

$\frac{\partial \varsigma}{\partial x}-A t \varsigma=\varphi(t)$

where $A=\frac{\rho g \sin \alpha}{4 \mu}$ and $\varphi(t)=1-A \int_{0}^{t} \varsigma_{1}(t) d t$

equation (60) is a first order differential equation whose general solution is given by

$\varsigma(x, t)=e^{A t x}\left[-\frac{\varphi(t)}{A t} e^{-A t x}+c\right]$

since at $x=0, \varsigma=0$ so from equation (61) we get $c=\varphi(t) / A t$, and then equation (61) gives

$\varsigma(x, t)=\left[\varphi(t) / A t\left(e^{A t x}-1\right)\right]$

Equation (57) and (62) gives

$h(x, t)=h_{0} \phi(t) e^{A t x}$

The solution of equation (63) is completed by specifying the boundary condition at the lower end of the film and here we consider only the case of a freely moving film of initial length $L_{\circ}$ and accordingly the suspended weight below a given material element remains constant in time, this means that $\varsigma_{1}=L_{0}$ and so equation (63), gives

$h(x, t)=h_{0}\left(1-A L_{0} t\right] e^{A t x}$

For drainage of a film on a frame with a fixed lower boundary as treated in this problem, the no flow condition requires that $u_{0}\left(L_{0}, t\right)=0$ and the general expression for the speed variation can be obtained from the profile equation:

$u_{0}(x, t)=-\frac{1}{h} \int_{0}^{x} \frac{\partial h(\eta, t)}{\partial t} d \eta$

equation (64) and (65), gives

$u_{0}(x, t)=\frac{1-e^{A t x}}{A t}\left(\frac{1}{t}-\frac{1}{f} \frac{d f}{d t}\right)-\frac{x}{t}$

It is necessary to note here that the condition $u_{0}\left(L_{0}, t\right)=0$ provides a new formula for the function $f(t)=1-A L_{0} t$ which appears in equation (64) subject to the condition $f(t) \rightarrow 1$ as $t \rightarrow 0$, and a closed expression for $f(t)$ can be found. From equation (66) and the condition $u_{0}\left(L_{0}, t\right)=0$, we get

$\int \frac{f^{\prime}(t)}{f(t)} d t=\int\left(\frac{1}{t}-\frac{A L_{0}}{1-e^{-A L_{0} t}}\right) d t$

which gives

$f(t)=\frac{e^{c} t}{e^{A L_{0} t}-1}$

As $f(t) \rightarrow 1$ as $t \rightarrow 0$, and from (67), we have $e^{c}=A L_{0}$ and so equation (67) becomes

$f(t)=\frac{A L_{0} t}{e^{A L_{0} t}-1}$

which is the formulation for $f(t)$ under the lower end boundary condition and thus equation (64) can be formulated by using equation (68) to give 
$h(x, t)=h_{0} L_{0} A t\left[e^{A L_{0} t}-1\right)^{-1} e^{A t x}$

Which is the required evolution for the thickness of the film.

Now we introduce the following non-dimensional variable:

$h=h_{0} h^{-}, x=L_{0} \bar{x}, t=\frac{3 \mu L_{0}^{4}}{\sigma_{0} h_{0}^{3}} t^{-}, h_{0}=\in L_{0}$

Now equation (69), gives

$h^{-}=L_{0} \frac{\rho g \sin \alpha}{4 \mu} \frac{3 \mu L_{0}^{4}}{\sigma h_{0}^{3}} t^{-}\left[e^{\frac{\rho g \sin \alpha}{4 \mu} \frac{3 \mu L_{0}^{4}}{\sigma h_{0}^{3} t^{-}}}-1\right]^{-1} e^{\frac{\rho g \sin \alpha}{4 \mu} \frac{3 \mu L_{0}^{4}}{\sigma h_{0}^{3}} t^{-} L_{0} \bar{x}}$

or

$h^{-}=C B_{1} t^{-}\left(e^{C B_{1} t^{-}}-1\right)^{-1} e^{C B_{1} t^{-} \bar{x}}$

where $C=\frac{3 \sin \alpha}{4 \epsilon^{2}}$

or

$B_{1}=\frac{B_{0}}{\in}$ (the modified Bond number).

By dropping tildes, equation (70), gives

$h(x, t)=\frac{C B_{1} t}{e^{C B_{1} t}-1} e^{B_{1} C t x}$

equation (71) is the non-dimensional form of equation (69).

Some of the solution curves are shown in figures (2), and (3) for different values of the inclination angle $\alpha$ and for different values of time.

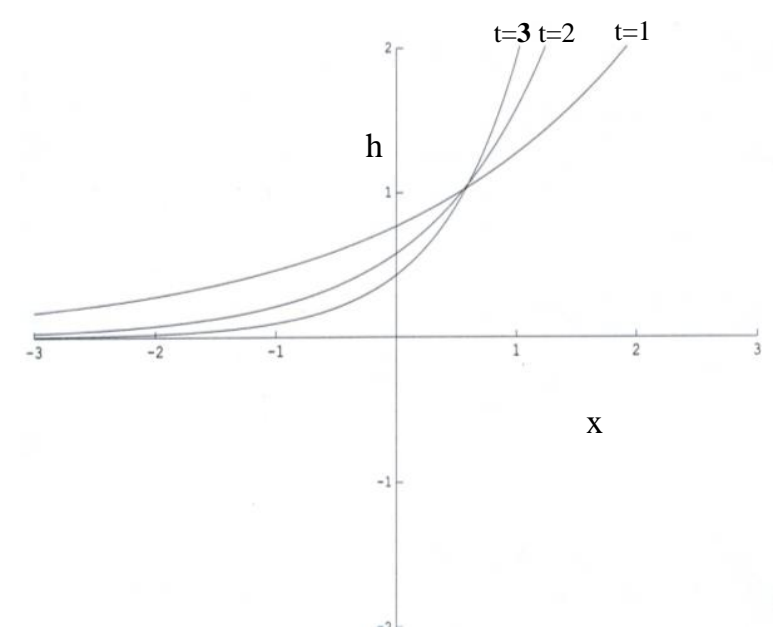

Figure (2): Draining film profile for $t=0, t=2 \mathrm{t}$ and $t=3$ inclination angle $\alpha=30^{\circ}$ and Bond number $B=0.1$.

$$
\alpha=60 \quad \alpha=30
$$




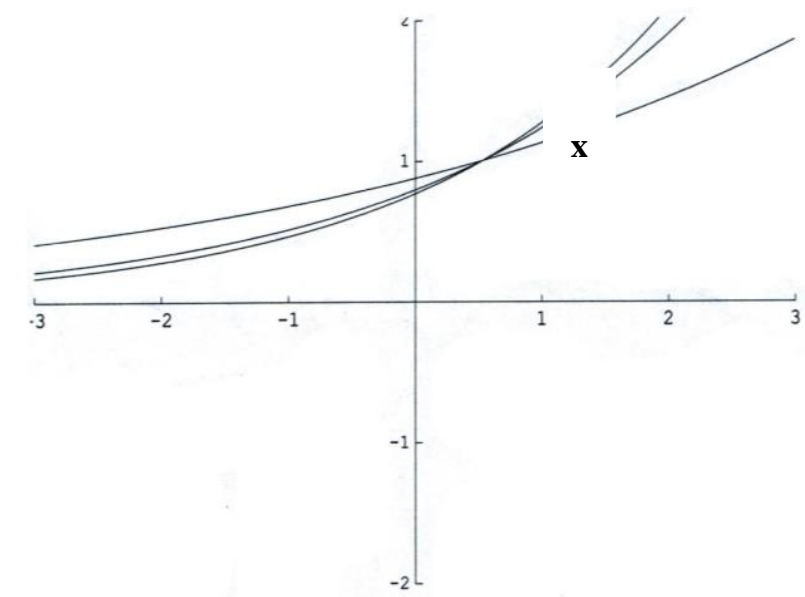

Figure (3): Draining film profile for $t=0.01 \mathrm{t}$ and inclination angles

$$
\alpha=30^{\circ}, 60^{\circ} \text { and } 90^{\circ} \text {. }
$$

\section{Conclusion:}

The film drainage reproduces different features that include the shape of the film profile and also in the draining time with or without surfactant effects. The film drains quickly when no surfactant exists and have a hollow ground appearance, but when the surfactant with high concentration exists, the film quickly look into an immobile interface with a parabolic shape that drains in slow manner and retains an appreciable thickness for long time and the film shape will be concaved out if the time drainage increases. 


\section{REFERENCE}

[1] Braun. R.J., Show. S.A and Prenisz. U.C. (1999). Gravitational drainage of a tangentially immobile thick film, Journal of Colloid and Interface Science, 219, 225-240.

[2] Davis S.H. and Bankef S.G. (1997). Long scale evolution of thin liquid films, Rev. Modern Phys., 69, 931-980.

[3] Kondic L. (2003). Instabilities in gravity driven flow of thin fluid films, Siam review, vol.45, No.1, pp.95-115.

[4] Leshansky, A. And Rubenstein, B. (2004). Nonlinear rupture of thin liquid films on solid surface, physics, vol.10.

[5] Myers T.G. (1998). Thin films with high surface tension, Siam Rev, 441-462.

[6] Myers T.G. (1996). Surface tension driven thin film flows, Word Scientific Publ. Constant. Pteltd.

[7] Mysels K., Shinoda K. and Frankel S. (1959). Soap film, Studies of their thinning, Pergamon, New York.

[8] Schwartz L.W. and Roy R.V. (1999). Modeling drainage flow in mobile and immobile soap films, Journal of Colloid and Interface Science, 218, 309-323./ 